\title{
CAMK4 gene variation is associated with hypertension in a Uygur population
}

\author{
S.Z. Chen ${ }^{1,2}$, Z. Wang ${ }^{1,2}$, L.N. Zhang ${ }^{1}$, G.L. Lu', C.M. Zhou', D.W. Wang', \\ Z.M. Tang ${ }^{3}$, L. Wang' ${ }^{2}$, L. Qin ${ }^{2}$ and Z.H. Zhai ${ }^{2}$ \\ 1Department of Internal Medicine and Genetic Diagnosis Center, Tongji Hospital, \\ Tongji Medical College, Huazhong University of Science and Technology, Wuhan, \\ Hubei, China \\ 2Department of Cardiology, First Affiliated Hospital, Shihezi Medical College, \\ Shihezi University, Shihezi, Xinjiang, China \\ ${ }^{3}$ Department of Cardiology, The People's Hospital of Tacheng, Tacheng, Xinjiang, \\ China
}

Corresponding author: Z. Wang

E-mail: wangzshz@163.com

Genet. Mol. Res. 15 (1): gmr.15017207

Received July 14, 2015

Accepted October 1, 2015

Published January 22, 2016

DOI http://dx.doi.org/10.4238/gmr.15017207

ABSTRACT. Considering that calcium/calmodulin-dependent kinase 4 (CAMK4) plays a pivotal role in blood pressure regulation, we investigated the association between a CAMK4 polymorphism (rs10491334) and hypertension in the Han, Kazak, and Uygur ethnic groups. We studied 1224 patients with hypertension and 967 normotensive controls classified into three ethnic groups (Han, Kazak, and Uygur). The rs10491334 polymorphism was genotyped using a $\operatorname{TaqMan}^{\circledR}{ }^{\circledR}$-nuclease assay. In the Uygur group, the T-allele frequency in patients with hypertension was twice that of the controls (12.5 vs $6.38 \%$ ), and T-allele carriers had a significantly increased risk of hypertension compared with non-carriers (odds ratio $=2.200 ; 95 \%$ confidence interval $=1.473-3.285, \mathrm{P}<0.001$ ) . However, no significant correlation was found in the Han and Kazak 
groups. The T-allele of rs10491334 in CAMK4 was associated with hypertension in the Uygur group.

Key words: Calcium/calmodulin-dependent kinase 4; CAMK4; rs10491334; Hypertension

\section{INTRODUCTION}

Hypertension, as a major cardiovascular disease risk factor, is a leading cause of morbidity and mortality worldwide, and places a major burden on individual and public health (Kearney et al., 2005). A large body of evidence bears out the rising interest in the contribution made by genetic factors to the development of hypertension. In particular, the most common form, essential hypertension $(E H)$, is widely believed to involve multiple genes with variant alleles (Levy et al., 2009; Newton-Cheh et al., 2009).

A genome-wide analysis of the Framingham Heart Study 100K Project in 2007 showed an association between elevated diastolic blood pressure (DBP) and a variant (rs10491334 T/C) of the calcium/calmodulin-dependent protein kinase 4 gene (CAMK4) (Levy et al., 2007). The CAMK4 gene encodes calcium/calmodulin-dependent protein kinase 4 and has the chromosomal locus $5 q 21.3$. The gene product belongs to the serine/threonine protein kinase family, and to the $\mathrm{Ca}^{2+} /$ calmodulin-dependent protein kinase subfamily. It is a multifunctional serine/threonine kinase enzyme with limited tissue distribution, and has been implicated in transcriptional regulation in lymphocytes, neurons, and male germ cells. Plenty of evidence indicates that CAMKs play a role in cardiovascular pathophysiology. For example, it is now clear that CAMK2 is an important player in the regulation of cardiac responses (Colomer et al., 2003; Zhang and Brown, 2004; Kushnir et al., 2010; Wagner et al., 2011), both in terms of electrophysiology and cardiac myocyte hypertrophy. However, the roles played by other members of the CAMK family in the cardiovascular system are less clear (Wayman et al., 2011).

In 2012, Santulli et al. reported that CAMK4 ${ }^{-/}$mice display a hypertensive phenotype that leads to typical organ damage. They indicated that CAMK4 plays an important role in the regulation of the vascular tone by a mechanism that involves eNOS activation through phosphorylative events. They also showed that in hypertensive patients, a CAMK4 polymorphism that causes reduced expression of the protein identifies a subset of patients with higher blood pressure (BP) levels.

On the basis of past studies, we conducted a case-control association analysis of the relationship between a CAMK4 polymorphism (rs10491334) and EH in 1217 hypertension patients and 963 normotensive controls recruited from Uygur, Kazak, and Han populations in the Xinjiang area of China.

\section{MATERIAL AND METHODS}

\section{Study design and participants}

This study included 1224 adults with $\mathrm{EH}$ (mean age: $55.90 \pm 11.23$ years). EH was defined as systolic blood pressure (SBP) ${ }^{3} 140 \mathrm{mmHg}$, orDBP $\geq 90 \mathrm{mmHg}$, or with a history of antihypertensive treatment after exclusion of secondary causes such as kidney disease. Unrelated Chinese volunteers without hypertension (967) were enrolled. The average age of the volunteers was $51.62 \pm 13.50$ years. All participants were from one of three ethnic groups (Han, Kazak, or 
Uygur), and had been admitted to Shihezi hospital in the Xinjiang Province of China for medical examination and routine treatment between January 2013 and December 2013. The present study was performed with the approval of the institutional review board of Shihezi hospital and is in compliance with the Helsinki Declaration. All participants provided written informed consent.

A questionnaire was completed that included complete demographic information (name, age, gender, smoking and drinking habits, history of disease, etc.). A complete general physical examination was carried out. BP was measured in a sitting position using a standard analog sphygmomanometer. Plasma glucose and lipid levels were measured using standard methods with an Olympus AU2700 automatic biochemical analyzer (Olympus Co., Ltd., Tokyo, Japan), after the patients had fasted for $12 \mathrm{~h}$ overnight.

\section{DNA samples and genotyping}

Each participant provided peripheral blood that was collected in 5-mL tubes containing ethylenediaminetetraacetic acid. Genomic DNA was extracted from the peripheral blood using a DNA isolation kit in accordance with the protocol (Tiangen Biotech, Beijing, China). The CAMK4 polymorphism (rs10491334) was genotyped using TaqMan ${ }^{\circledR}$ assays with a 7900 HT Fast Real-Time polymerase chain reaction (PCR) System (Applied Biosystems, Foster City, CA, USA). The reaction mixtures contained 2X TaqMan ${ }^{\circledR}$ universal PCR master mix (Applied Biosystems), 40X TaqMan ${ }^{\circledR}$ single $^{2}$ nucleotide polymorphism (SNP) genotyping assay (Applied Biosystems, Cat. \#4351379), and genomic DNA. The PCR cycling conditions comprised $50^{\circ} \mathrm{C}$ for $2 \mathrm{~min}, 95^{\circ} \mathrm{C}$ for $10 \mathrm{~min}$ followed by 45 cycles of $95^{\circ} \mathrm{C}$ for $15 \mathrm{~s}$, and $60^{\circ} \mathrm{C}$ for $1 \mathrm{~min}$. Genotype assignment was performed using the SDS software v2.3.

\section{Statistical analysis}

Statistical analysis was performed using SPSS17 (SPSS Inc., Chicago, IL, USA). Continuous variable data (age, SBP, DBP, waist-to-hip ratio, body mass index, glucose, triglyceride, total cholesterol, low-density lipoprotein cholesterol, high-density lipoprotein cholesterol, apolipoprotein A1, apolipoprotein B) are reported as means \pm standard deviation and were evaluated for normal distribution using the Kolmogorov-Smirnov test. Categorical variables (gender, smoking and drinking habits, history of coronary artery disease, stroke, diabetes, and hyperlipidemia) are shown as absolute numbers with prevalence. Differences in characteristics between patient and control groups were evaluated by independent-samples $t$-test and Pearson chi-square for continuous variables and categorical variables, respectively. Genotype and allele frequencies were calculated by the counting method. The Pearson chi-square test was employed to test whether the genotypes were in Hardy-Weinberg equilibrium, and was also used to compare the genotype and allele frequencies between the hypertension and control groups. Binary logistic regression was used to determine the association between rs10491334 and hypertension in three inheritance models: co-dominant, dominant, and recessive. All analyses were performed separately by race, and a double-sided $\mathrm{P}$ value $<0.05$ was considered to be statistically significant.

\section{RESULTS}

\section{Subject characteristics}

The characteristics of the hypertension and control groups are summarized in Table 1. In 
detail, SBP/DBP and waist-to-hip ratio were significantly higher in the hypertension group than in controls in all three ethnic groups. The mean age of hypertension was significantly higher in the Han and Kazak patients than in controls. There were no significant differences in gender between the hypertension and control groups. More hypertensive subjects had a history of diabetes and hyperlipidemia, but the laboratory data for glucose and lipid levels showed no significant differences between the groups.

\section{Genotype and allele frequencies of the CAMK4 polymorphism (rs10491334)}

The genotype and allele frequencies of the rs10491334 polymorphism are summarized in Table 2. The genotype distributions in the three ethnic groups were consistent with the HardyWeinberg equilibrium. In the Uygur group, the T-allele frequency in patients with hypertension was twice that of the controls (12.5 vs $6.38 \%)$. However, there were no significant differences in the genotype and allele frequencies in the other two ethnic groups, even after stratification analyses.

\section{Association analysis}

As shown in Table 3, association analysis revealed that T-allele carriers had a significantly increased risk of hypertension compared with non-carriers in the Uygur patients (odds ratio = 2.200; 95\% confidence interval $=1.473-3.285$, $\mathrm{P}<0.001$ ). However, no significant correlations were found in the Han and Kazak groups in three inheritance models.

Table 1. Baseline characteristics of the participants investigated.

\begin{tabular}{|c|c|c|c|c|c|c|}
\hline \multirow[t]{2}{*}{ Variables } & \multicolumn{2}{|c|}{ Han } & \multicolumn{2}{|c|}{ Kazak } & \multicolumn{2}{|c|}{ Uygur } \\
\hline & Hypertension & Controls & Hypertension & Controls & Hypertension & Controls \\
\hline Age (years) & $55.71 \pm 9.55^{*}$ & $46.10 \pm 13.41$ & $54.74 \pm 13.31^{*}$ & $49.15 \pm 14.94$ & $57.14 \pm 9.41$ & $56.06 \pm 10.33$ \\
\hline Male (\%) & $111(57.5)$ & $78(63.4)$ & $185(43.5)$ & $140(41.9)$ & $265(62.8)$ & 197 (58.1) \\
\hline $\mathrm{SBP}(\mathrm{mmHg})$ & $155.97 \pm 15.02^{*}$ & $119.54 \pm 10.39$ & $146.78 \pm 20.45^{\star}$ & $117.61 \pm 10.62$ & $149.18 \pm 18.35^{*}$ & $120.49 \pm 9.47$ \\
\hline $\mathrm{DBP}(\mathrm{mmHg})$ & $93.84 \pm 10.74^{*}$ & $75.89 \pm 6.76$ & $93.26 \pm 12.58^{*}$ & $75.58 \pm 7.61$ & $88.54 \pm 12.41^{*}$ & $76.27 \pm 5.97$ \\
\hline Waist-to-hip ratio & $0.93 \pm 0.05^{\star}$ & $0.90 \pm 0.05$ & $0.89 \pm 0.07^{\star}$ & $0.87 \pm 0.07$ & $0.90 \pm 0.06^{\star}$ & $0.86 \pm 0.04$ \\
\hline BMI $\left(\mathrm{kg} / \mathrm{m}^{2}\right)$ & $26.07 \pm 1.79^{*}$ & $24.84 \pm 2.74$ & $26.73 \pm 4.83^{*}$ & $24.42 \pm 3.84$ & $26.78 \pm 3.74$ & $27.07 \pm 4.52$ \\
\hline Smoking habit & $55(28.5)$ & $46(37.4)$ & $46(11.0)$ & $37(11.1)$ & $79(19.7)$ & 61 (19.1) \\
\hline Drinking habit & $68(35.2)$ & $51(41.5)$ & $16(11.0)$ & $13(18.3)$ & $9(7.8)$ & $5(7.8)$ \\
\hline History of CAD & $34(17.6)$ & $12(9.8)$ & $98(23.1)^{*}$ & $44(13.2)$ & $110(52.4)^{*}$ & $55(17.2)$ \\
\hline History of stroke & $5(2.6)$ & $2(1.6)$ & $16(3.8)$ & $5(1.5)$ & $17(4.2)$ & $7(2.2)$ \\
\hline History of diabetes & $31(16.1)^{*}$ & $3(2.4)$ & $4(2.8)$ & $2(2.8)$ & $33(28.4)^{*}$ & $1(10.9)$ \\
\hline History of hyperlipidemia & $61(31.6)^{*}$ & $23(18.7)$ & $73(17.4)^{*}$ & $20(6.0)$ & $126(31.4)^{*}$ & $79(24.8)$ \\
\hline Glucose (mM) & $5.77 \pm 2.44$ & $4.77 \pm 0.91$ & $5.19 \pm 1.31$ & $4.89 \pm 1.24$ & $6.07 \pm 2.73$ & $5.35 \pm 2.01$ \\
\hline Triglyceride (mM) & $1.77 \pm 1.25$ & $1.58 \pm 1.08$ & $1.29 \pm 0.90$ & $1.17 \pm 0.95$ & $1.87 \pm 1.11$ & $1.45 \pm 0.89$ \\
\hline Total cholesterol (mM) & $4.47 \pm 0.99$ & $4.35 \pm 0.85$ & $5.14 \pm 1.13$ & $4.71 \pm 1.02$ & $4.90 \pm 1.20$ & $4.52 \pm 1.09$ \\
\hline LDL-C (mM) & $2.57 \pm 0.75$ & $2.70 \pm 1.05$ & $2.89 \pm 0.74$ & $2.65 \pm 0.81$ & $3.19 \pm 1.08$ & $2.93 \pm 1.10$ \\
\hline HDL-C (mM) & $1.26 \pm 0.38$ & $1.22 \pm 0.29$ & $1.54 \pm 0.44$ & $1.48 \pm 0.47$ & $1.52 \pm 0.59$ & $1.59 \pm 0.56$ \\
\hline Apolipoprotein A1 (g/L) & $1.40 \pm 0.26$ & $1.41 \pm 0.29$ & $1.49 \pm 0.32$ & $1.40 \pm 0.31$ & $1.30 \pm 0.22$ & $1.32 \pm 0.22$ \\
\hline Apolipoprotein B (g/L) & $1.07 \pm 0.32$ & $1.01 \pm 0.22$ & $0.86 \pm 0.49$ & $0.74 \pm 0.24$ & $0.84 \pm 0.22$ & $0.77 \pm 0.18$ \\
\hline
\end{tabular}

SBP = systolic blood pressure; DBP = diastolic blood pressure; BMI = body mass index; CAD = coronary artery disease; LDL-C = low-density lipoprotein cholesterol; HDL-C = high-density lipoprotein cholesterol. Data are reported as means \pm SD for continuous variables and absolute numbers with prevalence for categorical variables. ${ }^{*} \mathrm{P}<0.05$ vs control, using independent-samples $t$-test for continuous variables and Pearson chi-square for categorical variable. 
Table 2. Genotype and allele frequencies of the calcium/calmodulin-dependent kinase 4 gene (CAMK4) polymorphism (rs10491334) in the Han, Kazak, and Uygur patients.

\begin{tabular}{|c|c|c|c|c|c|c|}
\hline Ethnicity & Population & $\mathrm{N}$ & MAF & $\mathrm{CC}$ & CT & TT \\
\hline \multirow[t]{2}{*}{ Han } & Controls & 290 & $7.76 \%$ & $247(85.2 \%)$ & $41(14.1 \%)$ & $2(0.7 \%)$ \\
\hline & Hypertension & 374 & $5.61 \%$ & $334(89.3 \%)$ & $38(10.2 \%)$ & $2(0.5 \%)$ \\
\hline \multirow[t]{2}{*}{ Kazak } & Controls & 336 & $10.42 \%$ & $266(79.2 \%)$ & $66(19.6 \%)$ & $4(1.2 \%)$ \\
\hline & Hypertension & 423 & $10.28 \%$ & $335(79.2 \%)$ & $83(19.6 \%)$ & $5(1.2 \%)$ \\
\hline \multirow[t]{2}{*}{ Uygur } & Controls & 337 & $6.38 \%$ & $297(88.1 \%)$ & $37(11.0 \%)$ & $3(0.9 \%)$ \\
\hline & Hypertension & 420 & $12.5 \%^{*}$ & $324(77.1 \%)$ & $87(20.7 \%)$ & $9(2.1 \%)$ \\
\hline \multirow[t]{2}{*}{ Total } & Controls & 963 & $8.41 \%$ & $810(84.1 \%)$ & $144(15.0 \%)$ & $9(0.9 \%)$ \\
\hline & Hypertension & 1217 & $9.86 \%$ & $993(81.6 \%)$ & $208(17.1 \%)$ & $16(1.3 \%)$ \\
\hline
\end{tabular}

MAF = minor allele frequency. ${ }^{*} \mathrm{P}<0.05$ vs control, using Pearson chi-square for allele frequencies.

Table 3. Odds ratios and $95 \%$ confidence intervals of calcium/calmodulin-dependent kinase 4 (CAMK4) to hypertension in Han, Kazak, and Uygur patients.

\begin{tabular}{llcccc}
\hline Inheritance model & Genotype & Han $(\mathrm{N}=664)$ & Kazak $(\mathrm{N}=759)$ & Uygur $(\mathrm{N}=757)$ & Total $(\mathrm{N}=2180)$ \\
\hline Co-dominant & CC & 1 & 1 & 1 & 1 \\
& CT & $0.740(0.103-5.286)$ & $0.993(0.264-3.733)$ & $2.750(0.737-10.254)$ & $1.450(0.637-3.299)$ \\
\multirow{2}{*}{ Dominant } & TT & $1.079(0.145-8.045)$ & $0.994(0.257-3.849)$ & $1.276(0.327-4.981)$ & $1.231(0.529-2.862)$ \\
& CC & 1 & 1 & 1 & 1 \\
Recessive & CT+TT & $0.688(0.434-1.091)$ & $0.998(0.701-1.420)$ & $2.200(1.473-3.285)^{\star}$ & $1.194(0.953-1.496)$ \\
& CC+CT & 1 & 1 & 1 & 1 \\
& TT & $0.774(0.108-5.529)$ & $0.993(0.265-3.726)$ & $2.438(0.655-9.077)$ & $1.412(0.621-3.210)$ \\
\hline
\end{tabular}

$\mathrm{P}<0.05$ using binary logistic regression.

\section{DISCUSSION}

CAMK4, previously known as a neuronal calmodulin-dependent multifunctional protein kinase (Kameshita and Fujisawa, 1991), occurs abundantly in the brain and is thought to be confined to the nervous system (Okuno and Fujisawa, 1993; Sakagami et al., 2000). In 2007, the Framingham Heart Study revealed an association marker for high DBP in the rs10491334 SNP of the human CAMK4 gene, suggesting that the kinase may have an as yet unidentified role in the control of blood pressure (Levy et al., 2007). Santulli et al. (2012) used a murine model derived from the genetic deletion of Camk4 (CaMK4 $4^{-r}$ ), which developed higher systolic and diastolic BP levels than its $\mathrm{CAMK}^{+/+}$littermates, and revealed the role of this kinase in hypertension. Malovini et al. (2011) found that homozygous carriers of rs10491334 displayed a significant reduction in CAMK4 expression, which was confirmed in Santulli et al.'s research (2012). These data were highly suggestive of the intrinsic regulatory nature of CAMK4 in hypertension.

Santulli et al. (2012) performed an association analysis with a candidate gene approach and found a significantly larger frequency of the T variant of the rs10491334 SNP in patients with severe hypertension than in patients with diastolic BP $<100 \mathrm{mmHg}(54.42$ vs $38.41 \%$; $\mathrm{P}<0.05$, Pearson chi-square analysis). The present study confirmed this finding in the Uygur group: the T-allele frequency in patients with hypertension was twice that in the controls ( $12.5 \mathrm{vs} 6.38 \%)$. We also showed that T-allele carriers had a significantly increased risk of hypertension compared with non-carriers (odds ratio $=2.200 ; 95 \%$ confidence interval $=1.473-3.285, \mathrm{P}<0.001$ ).

It is worth noting that the T-allele frequency in Europeans is significantly higher than that in Chinese, not only in patients but also in controls. Despite the fact that the three ethnic groups in the 
study were all Chinese, the research results differed markedly: the T-allele of rs10491334 in CAMK4 was associated with hypertension in the Uygur group, but there was no significant correlation in the Han and Kazak groups. This discrepancy could be attributed to genetic heterogeneity among these different populations. Some variants may be poor markers for hypertension because of differences in the linkage-disequilibrium structure in ethnic backgrounds (Wang et al., 2012). Differences in gene-gene or gene-environment interactions in the development of hypertension in these ethnicities may also contribute to the discrepancy.

In addition to hypertension, CAMK4 plays an important role in the development and progression of other cardiovascular diseases. Kato et al. (2000) reported that CAMK4 was critically involved in leukemia inhibitory factor-induced cardiac hypertrophy. Similarly, Passier et al. (2000) showed that activated $\mathrm{Ca}^{2+} /$ calmodulin-dependent protein kinases-1 and -4 (CAMK1 and CaMK4) also induced hypertrophic responses in cardiomyocytes in vitro, and that CAMK4-overexpressing mice developed cardiac hypertrophy with increased left ventricular end-diastolic diameter and decreased fractional shortening. In contrast, Colomer et al. (2003) found that mice null for the Camk4 gene developed ventricular hypertrophy and induced the expression of selected hypertrophy marker mRNAs, indicating that CAMK4 was not required at any time during the development of hypertrophy. Considering the inconsistent results, further research into the biological mechanism underlying the link between CAMK4 and cardiovascular diseases is required.

In summary, we verified a strong association between the CAMK4 polymorphism rs10491334 and hypertension in the Uygur population, but large-scale prospective cohort studies will be needed to confirm these findings.

\section{Conflicts of interest}

The authors declare no conflict of interest.

\section{ACKNOWLEDGMENTS}

Research supported by grants from the Projects Supporting Science and Technology at the Xinjiang Production and Construction Corps (\#2012AB014).

\section{REFERENCES}

Colomer JM, Mao L, Rockman HA and Means AR (2003). Pressure overload selectively up-regulates $\mathrm{Ca}^{2+} / \mathrm{calmodulin}^{-}$ dependent protein kinase II in vivo. Mol. Endocrinol. 17: 183-192.http://dx.doi.org/10.1210/me.2002-0350

Kameshita I and Fujisawa $\mathrm{H}$ (1991). Phosphorylation and functional modification of calmodulin-dependent protein kinase IV by cAMP-dependent protein kinase. Biochem. Biophys. Res. Commun. 180: 191-196. http://dx.doi.org/10.1016/S0006-291X(05)81275-6

Kato T, Sano M, Miyoshi S, Sato T, et al. (2000). Calmodulin kinases II and IV and calcineurin are involved in leukemia inhibitory factor-induced cardiac hypertrophy in rats. Circ. Res. 87: 937-945.http://dx.doi.org/10.1161/01.RES.87.10.937

Kearney PM, Whelton M, Reynolds K, Muntner P, et al. (2005). Global burden of hypertension: analysis of worldwide data. Lancet 365: 217-223.http://dx.doi.org/10.1016/S0140-6736(05)70151-3

Kushnir A, Shan J, Betzenhauser MJ, Reiken S, et al. (2010). Role of CaMKIldelta phosphorylation of the cardiac ryanodine receptor in the force frequency relationship and heart failure. Proc. Natl. Acad. Sci. USA 107: 10274-10279. http://dx.doi.org/10.1073/pnas.1005843107

Levy D, Larson MG, Benjamin EJ, Newton-Cheh C, et al. (2007). Framingham Heart Study 100K Project: genome-wide associations for blood pressure and arterial stiffness. BMC Med. Genet. 8 (Suppl 1): S3. http://dx.doi.org/10.1186/1471-2350-8-S1-S3 
Levy D, Ehret GB, Rice K, Verwoert GC, et al. (2009). Genome-wide association study of blood pressure and hypertension. Nat. Genet. 41: 677-687.http://dx.doi.org/10.1038/ng.384

Malovini A, Illario M, laccarino G, Villa F, et al. (2011). Association study on long-living individuals from Southern Italy identifies rs10491334 in the CAMKIV gene that regulates survival proteins. Rejuvenation Res. 14: 283-291.http://dx.doi. org/10.1089/rej.2010.1114

Newton-Cheh C, Johnson T, Gateva V, Tobin MD, et al.; Wellcome Trust Case Control Consortium (2009). Genome-wide association study identifies eight loci associated with blood pressure. Nat. Genet. 41: 666-676. http://dx.doi.org/10.1038/ng.361

Okuno $\mathrm{S}$ and Fujisawa $\mathrm{H}$ (1993). Requirement of brain extract for the activity of brain calmodulin-dependent protein kinase IV expressed in Escherichia coli. J. Biochem. 114: 167-170.

Passier R, Zeng H, Frey N, Naya FJ, et al. (2000). CaM kinase signaling induces cardiac hypertrophy and activates the MEF2 transcription factor in vivo. J. Clin. Invest. 105: 1395-1406.http://dx.doi.org/10.1172/JCI8551

Sakagami H, Umemiya M, Saito S and Kondo H (2000). Distinct immunohistochemical localization of two isoforms of Ca2+/ calmodulin-dependent protein kinase kinases in the adult rat brain. Eur. J. Neurosci. 12: 89-99. http://dx.doi.org/10.1046/j.1460-9568.2000.00883.x

Santulli G, Cipolletta E, Sorriento D, Del Giudice C, et al. (2012). CaMK4 Gene Deletion Induces Hypertension. J. Am. Heart Assoc. 1: e001081.http://dx.doi.org/10.1161/JAHA.112.001081

Wagner S, Ruff HM, Weber SL, Bellmann S, et al. (2011). Reactive oxygen species-activated Ca/calmodulin kinase IIס is required for late I(Na) augmentation leading to cellular $\mathrm{Na}$ and $\mathrm{Ca}$ overload. Circ. Res. 108: 555-565. http://dx.doi.org/10.1161/CIRCRESAHA.110.221911

Wang Z, Xu Y, Chen S, Wang L, et al. (2012). A common missense single nucleotide polymorphism in the E-selectin gene is significantly associated with essential hypertension in the Han population but only weakly associated in the Uygur population. Hypertens. Res. 35: 413-417.http://dx.doi.org/10.1038/hr.2011.204

Wayman GA, Tokumitsu H, Davare MA and Soderling TR (2011). Analysis of CaM-kinase signaling in cells. Cell Calcium 50: 1-8.http://dx.doi.org/10.1016/j.ceca.2011.02.007

Zhang T and Brown JH (2004). Role of $\mathrm{Ca}^{2+} /$ calmodulin-dependent protein kinase II in cardiac hypertrophy and heart failure. Cardiovasc. Res. 63: 476-486.http://dx.doi.org/10.1016/j.cardiores.2004.04.026 